\title{
Associations between maternal healthy lifestyle score and offspring birth outcomes and childhood obesity: results from the Lifeways Cross-Generation Cohort Study
}

\author{
Pilar Navarro, John Mehegan, Celine Murrin, Cecily Kelleher and Catherine Phillips \\ University College Dublin, Dublin, Ireland
}

\section{Abstract}

Maternal adherence to healthy lifestyle behaviours during pregnancy has been associated with reduced risk of obesity in the offspring. Our objective is to examine the association between a composite healthy lifestyle score (HLS) derived from body mass index (BMI), dietary quality, physical activity, smoking and alcohol intake, in expectant mothers and adverse offspring birth outcomes and childhood obesity. The Lifeways Cross-Generation Study comprises 1082 mother-child pairs. We defined five healthy lifestyle factors during pregnancy including: high dietary quality (top 40\% of the Healthy Eating Index (HEI)-2015), moderate to vigorous physical activity (MVPA), healthy pre-pregnancy BMI $\left(18.5-24.9 \mathrm{Kg} / \mathrm{m}^{2}\right)$, never smoker, and no/moderate alcohol intake. A composite HLS was calculated (scored 0-5). Birthweight, length and head circumference were abstracted from hospital records. Waist circumference and BMI was determined when the child was 5 and 9 years. Logistic regression analyses were used to test HLS and individual HLS component associations with offspring birth and childhood outcomes. Offspring birthweight, length and head circumference were positively associated with the number of maternal healthy lifestyle factors $(p<0.001)$, whereas child BMI and incidence of overweight/obesity at age 5 and 9 were negatively associated with the maternal HLS $(p<0.05)$. In multivariable models, a lower maternal HLS (0-2 healthy lifestyle factors) was associated with increased risk of low birthweight (LBW) (OR:1.17, 95\% CI:1.01-2.69, $p=$ 0.043 ) and lower likelihood of macrosomia (OR:0.73, 95\% CI:0.24-0.99, $p=0.034$ ), relative to those with 5 healthy lifestyle factors. Examination of the individual HLS components revealed that, poor maternal dietary quality, smoking and alcohol intake were associated with higher risk of LBW (OR:1.61, 95\%CI:1.01-7.85, $p=0.043$, OR: 2.54, 95\%CI:1.26-5.12, $p=0.025$ and OR:2.30, 95\% CI:1.01-5.26, $p=0.031$, respectively). Likelihood of macrosomia and combined overweight/obesity at age 5 and 9 years was greater among mothers with a pre-pregnancy BMI in the obese range (OR:2.18, 95\%CI:1.23-3.85, $p=0.042, \mathrm{OR}: 2.19,95 \% \mathrm{CI}: 1.01-5.08, p=$ 0.03 and OR:3.89, 95\%CI:1.00-10.59, $p=0.04$, respectively). Smoking during pregnancy was also linked to greater risk of childhood overweight/obesity (OR:1.91, 95\%CI:1.01-3.61, $p=0.04$ at age 5 and OR:2.14, 95\%CI:1.01-4.11, $p=0.03$ at age 9). Our findings suggest that maternal adherence to a healthy lifestyle during pregnancy, in particular having a good quality diet, not smoking and no/low alcohol intake in combination with a healthy pre-pregnancy BMI, is associated with reduced risk of adverse offspring birth outcomes and childhood obesity. These findings highlight the potential benefits of implementing maternal based multifactorial interventions to improve offspring birth outcomes and combat childhood adiposity.

\section{Conflict of Interest}

There is no conflict of interest 